\title{
CIRCULAR DISTRIBUTIONS OF FINITE ORDER
}

\author{
SoOGIL SEO
}

\begin{abstract}
Motivated by the theory of circular distributions, we introduced a filtration on the global units attached to the maximal real subfield of a cyclotomic field and conjectured that the associated gradation is isomorphic, (as a Galois module) to the ideal class group. This conjecture depends on circular distributions of Coleman and a guess made by him. In this paper we show that the circular distributions of finite order cannot be constructed from cyclotomic p-units and their Galois conjugates.
\end{abstract}

\section{Introduction}

This paper concerns circular distributions, that is, Galois equivariant maps from the roots of unity to the algebraic numbers that satisfy the same distribution relation as the roots of unity themselves.

A natural class of circular distributions can be formed from cyclotomic punits and their Galois conjugates. Coleman in the 80 s made a guess that certain congruence conditions characterize this special class of distributions(cf. [8], [9]), and this paper shows that Coleman's guess is not quite right. We present a counterexample in the form of a distribution that takes only the values 1 and -1 , and give an explicit calculation showing that this example cannot be constructed in the way alluded to above.

The circular distributions which arise in various contexts in number theory specially give rise to Euler systems and special units over number fields. It is known that the order of the class group $\mathrm{Cl}_{K}$ of real abelian field $\mathrm{K}$ is essentially equal to the order of the quotient $E_{K} / C_{K}$ of the units of $\mathrm{K}$ by the circular units of $\mathrm{K}$, but the group structures of these two groups are usually very different. Motivated by the theory of circular distributions and that of the special units of Rubin, we introduced a filtration on $E_{K}$ as a Galois module and conjectured that the associated gradation is isomorphic to $\mathrm{Cl}_{K}$ (as a Galois module). This conjectural description of the ideal class groups in terms of a special unit filtration depends heavily on Coleman's modified guess stated below.

Let $\mu_{n}$ denote the group of $n$-th roots of unity in a fixed algebraic closure $\mathbb{Q}^{\text {alg }}$ of $\mathbb{Q}$ and $\mu_{n}^{*}$ the subset of $\mu_{n}$ containing all roots of unity which are not

Received by the editors April 15, 2004.

2000 Mathematics Subject Classification. 11R18,11R23, 11R27, 11R29.

This work was supported in part by Yonsei University Reasearch Fund of 2005. 
equal to one. Let $\mu_{\infty}^{*}$ denote the union of all subsets $\mu_{n}^{*}$. A circular distribution is a Galois equivariant map $f$ from $\mu_{\infty}^{*}$ to $\mathbb{Q}^{\text {alg }}$ such that for any $\epsilon$ in $\mu_{\infty}^{*}$ and positive integer $d$, it satisfies the following distribution property,

$$
\prod_{\zeta^{d}=\epsilon} f(\zeta)=f(\epsilon)
$$

where the product is taken over all $\zeta$ with $\zeta^{d}=\epsilon$.

We denote by $\Sigma$ the set of all circular distributions. Let $R$ denote the projective limit $\lim \mathbb{Z}\left[\operatorname{Gal}\left(\mathbb{Q}\left(\mu_{n}\right) / \mathbb{Q}\right)\right]$ of the group ring $\mathbb{Z}\left[\operatorname{Gal}\left(\mathbb{Q}\left(\mu_{n}\right) / \mathbb{Q}\right)\right]$, where the inverse limit is taken over the natural restriction maps from $\operatorname{Gal}\left(\mathbb{Q}\left(\mu_{m}\right) / \mathbb{Q}\right)$ to $\operatorname{Gal}\left(\mathbb{Q}\left(\mu_{n}\right) / \mathbb{Q}\right)$ for each $m \geq n$. Then $\Sigma$ has a natural $R$ module structure. Among all circular distributions, most crucial and natural is the following distribution

$$
\phi(\zeta)=1-\zeta, \quad \zeta \in \mu_{\infty}^{*} .
$$

The first question, due to Coleman, is whether the set of circular distributions is a cyclic $R$ module generated by $\phi$. By finding examples(see below) he imposes the following congruence relations to $f \in \Sigma$; for each prime number $l$ and $n \in \mathbb{N}$ with $(l, n)=1$,

$$
f(\epsilon \zeta) \equiv f(\zeta) \text { modulo primes over }(l) \text { for all } \epsilon \in \mu_{l}^{*}, \zeta \in \mu_{n}^{*} .
$$

We denote by $\mathcal{F}$ the $R$ submodule of $\Sigma$ satisfying the above congruence relations. Let $R_{n}$ be the group $\operatorname{ring} \mathbb{Z}\left[\operatorname{Gal}\left(\mathbb{Q}\left(\mu_{n}\right) / \mathbb{Q}\right)\right]$. Let $\zeta_{n}$ denote a fixed generator of the group $\mu_{n}$. For an element $\left(r_{n}\right)$ in the product $\prod R_{n}$, we define $\psi_{\left(r_{n}\right)}$ to be $\psi_{\left(r_{n}\right)}\left(\zeta_{n}\right):=\left(1-\zeta_{n}\right)^{r_{n}}$. Let $\Psi$ denote the set $\left\{\psi_{r} \mid r \in R\right\}$. We recall Coleman's guess on circular distributions that $\mathcal{F}$ is a cyclic $R$-module generated by $\psi$ :

\section{Does $\mathcal{F}$ equal $\Psi$ ?}

The following example is due to Coleman. For any set $S$ of odd primes, let $\delta_{S}$ be the circular distribution whose value at $\zeta_{n}$ is either -1 or 1 , according as $n$ involves only primes in $S$ or not. Let $\mathcal{D}$ be the $R$-submodule of $\Sigma$ generated by $\delta_{S}$ for all such $S$. When $S$ is the set of all odd primes, we denote $\delta_{S}$ by $\delta_{\text {odd }}$. In [10], we computed the torsion subgroups $\Sigma_{\text {tor }}$ and $\mathcal{F}_{\text {tor }}$ of $\Sigma$ and $\mathcal{F}$ respectively: $\Sigma_{\text {tor }}=\mathcal{D}, \mathcal{F}_{\text {tor }}=\left\{1, \delta_{\text {odd }}\right\}$.

In Section 2 we compute the $\mathbb{Z}$-torsion subgroup $\Psi_{\text {tor }}$ of $\Psi$. It follows from $\Psi_{\text {tor }} \subset \mathcal{F}_{\text {tor }}$ that $\Psi_{\text {tor }} \subset\left\{1, \delta_{\text {odd }}\right\}$. By showing that $\delta_{\text {odd }}$ is not contained in $\Psi$ we will show that $\Psi$ is a free $\mathbb{Z}$-module. This shows that $\mathcal{F}_{\text {tor }}$ differs from $\Psi_{\text {tor }}$ giving a negative answer to Coleman's guess.

Theorem A. $\mathcal{F} \neq \Psi$. 
Theorem A lead us to a natural modification, an affirmative answer to which would be a slight modification of Coleman's original guess on the circular distributions:

Does $\mathcal{F}$ equal $\Psi \oplus \mathcal{F}_{\text {tor }}$ ?

As we mentioned, one of important applications of the question above is on the Galois module structures of the ideal class groups of abelian fields. The circular distributions are naturally used to define the notion of the higher special units for abelian fields.

We briefly introduce the definition of higher special units of an abelian number field $K$. Let $K$ be an abelian extension of $F$ containing the Hilbert class field of $F$. Let $I_{K / F}^{r}$ be the set of square free integral fractional ideals $\mathfrak{a}$ such that each prime $\mathfrak{p}$ dividing $\mathfrak{a}$ has absolute degree one and splits completely in $K$ and the number of primes dividing $\mathfrak{a}$ is less than or equal to $r$. For each prime ideal $\mathfrak{p}$ of $F$, let $F(\mathfrak{p})$ denote the ray class field of $F$ modulo $\mathfrak{p}$ and for an integral fractional ideal $\mathfrak{a}, F(\mathfrak{a})$ the composite field $\prod_{\mathfrak{p} \mid \mathfrak{a}} F(\mathfrak{p})$. Let $K(\mathfrak{a})$ be the composite field of $K$ and $F(\mathfrak{a})$. Write $w(p)$ for the order of the image of $E_{F}$ in $\left(\mathcal{O}_{F} / p\right)^{\times}$, where $\mathcal{O}_{F}$ denotes the ring of integers of $F$. Let $\mathcal{E}_{K / F}^{r}$ be the set of maps $\psi$ from $I_{K / F}^{r}$ to a fixed algebraic closure $F^{\text {alg }}$ such that for each $\mathfrak{a} \in I_{K / F}^{r}$ and prime $\mathfrak{p} \mid \mathfrak{a}$,

$$
\psi(\mathfrak{a}) \in E_{F(\mathfrak{a})}, \quad N_{K(\mathfrak{a}) / K\left(\mathfrak{a} \mathfrak{p}^{-1}\right)} \psi(\mathfrak{a})=\psi\left(\mathfrak{a p}^{-1}\right)^{F r_{p}-1}
$$

and $\psi(\mathfrak{a}) \equiv \psi\left(\mathfrak{a p}^{-1}\right)^{w(p)}$ modulo primes over $\mathfrak{p}$. Following Rubin's definition(cf. [6]) of special units, we define the special units $S_{K / F}^{r}$ of level $r$ to be $\left\{\psi\left(\mathcal{O}_{F}\right) \mid \psi \in \mathcal{E}_{K / F}^{r}\right\}$. When the ground field $F$ is equal to $\mathbb{Q}$ we write $\mathcal{E}_{K}^{r}$ and $S_{K}^{r}$ respectively for $\mathcal{E}_{K / F}^{r}$ and $S_{K / F}^{r}$. Using the class number formula(cf. [12]) we compare the group structure of $\mathrm{Cl}_{K}$ with that of a filtration of higher special units and propose a conjecture whether they are isomorphic when $K$ is a real abelian field. Let $h_{K}$ denote the class number of $K$.

Conjecture. Suppose $K$ is a real abelian field and $p$ an odd prime with $p \nmid$ $\left(h_{K},[K: \mathbb{Q}]\right)$. Then there is the following $\mathbb{Z}_{p}[\operatorname{Gal}(K / \mathbb{Q})]$ module isomorphism,

$$
\mathrm{Cl}_{K} \otimes \mathbb{Z}_{p} \simeq \bigoplus_{n \geq 0}\left(\frac{S_{K}^{n}}{S_{K}^{n+1}} \otimes \mathbb{Z}_{p}\right) .
$$

Hence if we let $\mathbb{Z}^{\prime}$ denote the ring obtained by adding to $\mathbb{Z} p^{-1}$ for $p \mid 2\left(h_{K},[K\right.$ : $\mathbb{Q}])$ then then there is the following $\mathbb{Z}^{\prime}[\mathrm{Gal}(K / \mathbb{Q})]$ module isomorphism,

$$
\mathrm{Cl}_{K} \simeq \bigoplus_{n \geq 0} \frac{S_{K}^{n}}{S_{K}^{n+1}}
$$

In his paper [4], Kolyvagin made a theorem(=Theorem 7 of [4]) on the description of the structures of both the Selmer groups and the class groups of abelian fields in terms of certain sets of primes. His arguments gives bases of 
the class groups and the Selmer groups. For a similar structure theorem on the description of certain cohomology groups in terms of filtration of certain quotients of a ring which is more general can be found in a theorem of Mazur and $\operatorname{Rubin}(=$ Theorem 4.5 .9 of $[5])$.

The conjectural description above depends entirely on Coleman's modified guess and class number formula(cf. [12]) in order to get the equality on the cardinalities of both objects. We used Euler systems(cf. [7]) to give evidence for this conjecture. For the details we refer the reader to [11] or a subsequent paper.

In Section 3 we investigate norm coherence circular $p$-units over $\mathbb{Z}_{p}$ extensions. We show that for any prime $p$ the inverse limit $\left\{\delta_{\text {odd }}\left(\zeta_{p^{n}}\right)\right\}$ of circular distribution $\delta_{\text {odd }}$ is equal to the inverse limit $\left\{\psi_{r}\left(\zeta_{p^{n}}\right)\right\}$ for some $r \in R$ over the basic $\mathbb{Z}_{p}$ extension.

Finally we will give a guess on a necessary and sufficient condition for a norm coherence sequence to be a circular $p$-units(cf. [1] and [2]).

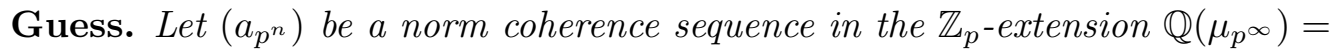
$\bigcup \mathbb{Q}\left(\mu_{p^{n}}\right)$. Then $a_{p^{n}}$ is a norm coherence sequence of circular $p$-units if and only if there exists an $f \in \mathcal{F}$ such that $f\left(\zeta_{p^{n}}\right)=a_{p^{n}}$ for all $n$.

\section{Main results}

We recall notations defined in the previous section. Let $\mu_{n}$ be the group of $n$-th roots of unity in $\mathbb{Q}^{\text {alg }}$ and let $\mu_{n}^{*}=\mu_{n} \backslash\{1\}$. Let $\mu_{\infty}=\bigcup_{n \in \mathbb{N}} \mu_{n}$ and $\mu_{\infty}^{*}=$ $\mu_{\infty} \backslash\{1\}$. Let $\zeta_{n}$ be a primitive $n$-th roots of unity in $\mu_{n}$ such that $\zeta_{m n}^{m}=\zeta_{n}$ for all $m, n \in \mathbb{N}$. As defined in the introduction let $R_{n}=\mathbb{Z}\left[\operatorname{Gal}\left(\mathbb{Q}\left(\mu_{n}\right) / \mathbb{Q}\right)\right]$ be the group ring of the Galois group $\operatorname{Gal}\left(\mathbb{Q}\left(\mu_{n}\right) / \mathbb{Q}\right)$ and $R=\lim _{\longleftarrow} R_{n}$ be the projective limit of $R_{n}$ with respect to the natural restriction maps. For a sequence $\left(r_{n}\right) \in \prod R_{n}$, we define $\psi_{\left(r_{n}\right)}$ to be $\psi_{\left(r_{n}\right)}\left(\zeta_{n}\right)=\left(1-\zeta_{n}\right)^{r_{n}}$. Let $\Psi=\left\{\psi_{r} \mid r \in R\right\}$.

In this section we will compute the $\mathbb{Z}$-torsion $\Psi_{\text {tor }}$ of $\Psi$. We need to recall the torsion parts $\mathcal{F}_{\text {tor }}$ and $\Psi_{\text {tor }}$ of $\mathcal{F}$ and $\Psi$ respectively. Let $S$ be a set of odd primes. As in the introduction we let $\delta_{S}$ be the function on $\mu_{\infty}^{*}$ defined to be

$$
\delta_{S}\left(\zeta_{n}\right)= \begin{cases}-1 & \text { if } \mathrm{n} \text { involves only primes in } S \\ 1 & \text { otherwise. }\end{cases}
$$

We let $\mathcal{D}$ be the $R$-submodule of $\Sigma$ generated by $\delta_{S}$ for all such $S$. When $S$ is the set of all odd primes, we denote $\delta_{S}$ by $\delta_{\text {odd }}$. The following theorem was proved.

Theorem 2.1. $\Sigma_{\text {tor }}=\mathcal{D}, \mathcal{F}_{\text {tor }}=\left\{1, \delta_{\text {odd }}\right\}$.

Proof. See [9].

We will use the following isomorphisms of Galois groups: Let $m$ be an odd number and let $\mathbb{Q}\left(\mu_{4 m}\right)^{+}:=\mathbb{Q}\left(\zeta_{4 m}+\zeta_{4 m}^{-1}\right)$ be the maximal real subfield of $\mathbb{Q}\left(\mu_{4 m}\right)$. Then there are natural isomorphisms

$$
\operatorname{Gal}\left(\mathbb{Q}\left(\mu_{4 m}\right) / \mathbb{Q}\left(\mu_{4}\right)\right) \simeq \operatorname{Gal}\left(\mathbb{Q}\left(\mu_{m}\right) / \mathbb{Q}\right) \simeq \operatorname{Gal}\left(\mathbb{Q}\left(\mu_{4 m}\right)^{+} / \mathbb{Q}\right)
$$


The complex conjugation $\tau \in \operatorname{Gal}\left(\mathbb{Q}\left(\mu_{4}\right) / \mathbb{Q}\right)$ acts trivially on $\operatorname{Gal}\left(\mathbb{Q}\left(\mu_{4 m}\right) / \mathbb{Q}\left(\mu_{4}\right)\right)$. Thus we will see $\mathbb{Z}\left[\operatorname{Gal}\left(\mathbb{Q}\left(\mu_{4 m}\right) / \mathbb{Q}\left(\mu_{4}\right)\right)\right]$ as a

$$
\mathbb{Z}\left[\operatorname{Gal}\left(\mathbb{Q}\left(\mu_{4 m}\right) / \mathbb{Q}\right)\right]=\mathbb{Z}\left[\operatorname{Gal}\left(\mathbb{Q}\left(\mu_{m}\right) / \mathbb{Q}\right)\right] \otimes \mathbb{Z}\left[\operatorname{Gal}\left(\mathbb{Q}\left(\mu_{4}\right) / \mathbb{Q}\right)\right] \text {-module. }
$$

Let $C_{n}$ be the circular units of $\mathbb{Q}\left(\mu_{n}\right)$, i.e., the subgroup of $E_{\mathbb{Q}\left(\mu_{n}\right)}$ generated over $\mathbb{Z}\left[\operatorname{Gal}\left(\mathbb{Q}\left(\mu_{n}\right) / \mathbb{Q}\right)\right]$ by $1-\zeta$ as $\zeta$ runs through all $n$-th roots of unity. Let $D_{n}:=\left(1-\zeta_{n}\right)^{R_{n}}$ be the subgroup of $C_{n}$ generated by $\left(1-\zeta_{n}\right)$ over $R_{n}$.

We assume from now on that the $\mathbb{Z}$-rank of $D_{n}$ is equal to the $\mathbb{Z}$-rank of the group of units. This is the case if the $\mathbb{Z}$ rank of $D_{n}$ equals the $\mathbb{Z}$ rank of the group of circular units. Note that if $n$ is a prime power then this is always true. We denote by $N_{+}(n)$ the norm map from $\mathbb{Q}\left(\mu_{n}\right)^{+}$to $\mathbb{Q}$;

$$
N_{+}(n):=\sum_{\sigma \in \operatorname{Gal}\left(\mathbb{Q}\left(\mu_{n}\right)^{+} / \mathbb{Q}\right)} \sigma .
$$

First let $n$ be not a prime power. We denote by $\left(D_{n}\right)_{\text {tor }}$ the $\mathbb{Z}$-torsion part of $D_{n}$. The map $\rho$ defined as $\rho\left(\sum a_{\sigma} \sigma\right):=\left(1-\zeta_{4 n}\right)^{\sum a_{\sigma} \sigma}$ gives us the following surjection,

$$
\frac{\mathbb{Z}\left[\operatorname{Gal}\left(\mathbb{Q}\left(\mu_{n}\right)^{+} / \mathbb{Q}\right)\right]}{<N_{+}(n)>} \longrightarrow D_{n} /\left(D_{n}\right)_{\text {tor }} \longrightarrow 0
$$

Dirichlet's unit theorem shows they have the same $\mathbb{Z}$-ranks. Notice that the complex conjugation $\tau$ acts trivially on the left side and $\tau$ also acts trivially on the right side as

$$
(\tau-1) D_{n} \subset\left(D_{n}\right)_{\text {tor }} .
$$

Moreover these two are torsion free $\mathbb{Z}\left[\operatorname{Gal}\left(\mathbb{Q}\left(\mu_{n}\right) / \mathbb{Q}\right)\right]$-modules. Thus it follows that the surjection above is an isomorphism.

If $n$ is a prime power $p^{r}$ then $D_{n}$ is not contained in the group of units anymore. It is the group of $p$-units which has the same $\mathbb{Z}$-rank as $\mathbb{Z}\left[\operatorname{Gal}\left(\mathbb{Q}\left(\mu_{n}\right)^{+} / \mathbb{Q}\right)\right]$. Moreover $D_{n} /\left(D_{n}\right)_{\text {tor }}$ and $\mathbb{Z}\left[\operatorname{Gal}\left(\mathbb{Q}\left(\mu_{n}\right)^{+} / \mathbb{Q}\right)\right]$ are torsion free and hence $\rho$ induces the following isomorphism,

$$
D_{n} /\left(D_{n}\right)_{\text {tor }} \simeq \mathbb{Z}\left[\operatorname{Gal}\left(\mathbb{Q}\left(\mu_{n}\right)^{+} / \mathbb{Q}\right)\right]
$$

We have proved the following Lemma.

Lemma 2.2. Suppose that $D_{n}$ has the same $\mathbb{Z}$ rank as that of the group of circular units when $n$ is not a prime power. As $\mathbb{Z}\left[\operatorname{Gal}\left(\mathbb{Q}\left(\mu_{4 n}\right) / \mathbb{Q}\right)\right]$-modules, we have isomorphisms

$$
D_{n} /\left(D_{n}\right)_{\text {tor }} \stackrel{\sim}{\longrightarrow} \begin{cases}\mathbb{Z}\left[\operatorname{Gal}\left(\mathbb{Q}\left(\mu_{n}\right)^{+} / \mathbb{Q}\right)\right] & \text { if } n \text { is a prime power }, \\ \mathbb{Z}\left[\operatorname{Gal}\left(\mathbb{Q}\left(\mu_{n}\right)^{+} / \mathbb{Q}\right)\right] /<N_{+}(n)> & \text { otherwise. }\end{cases}
$$

For an $R$-module $M$, we denote by $\operatorname{ann}_{R} M$ the annihilator of $M$ in $R$,

$$
\operatorname{ann}_{R} M:=\{r \in R \mid r \cdot m=0\} .
$$

For each $\sigma \in \operatorname{Gal}\left(\mathbb{Q}\left(\mu_{r}\right) / \mathbb{Q}\right)$ we write $\sigma=\sigma_{i(r)}$ if $\sigma\left(\zeta_{r}\right)=\zeta_{r}^{i(r)}$. Note that the subscript $i(r)$ in $\sigma=\sigma_{i(r)}$ depends on $r$. We will write $i(r)=i$ if no 
confusion occurs in the context. From the following $\mathbb{Z}\left[\operatorname{Gal}\left(\mathbb{Q}\left(\mu_{4 n}\right) / \mathbb{Q}\right)\right]$-module isomorphism

$$
\frac{\mathbb{Z}\left[\operatorname{Gal}\left(\mathbb{Q}\left(\mu_{n}\right)^{+} / \mathbb{Q}\right)\right]}{<N_{+}>} \stackrel{\sim}{\longrightarrow} \frac{\mathbb{Z}\left[\operatorname{Gal}\left(\mathbb{Q}\left(\mu_{n}\right) / \mathbb{Q}\right)\right]}{<\sigma_{i}-\sigma_{-i} \mid \sigma_{i} \in \operatorname{Gal}\left(\mathbb{Q}\left(\mu_{n}\right) / \mathbb{Q}\right), N_{+}>}
$$

if $n$ is not a prime power and

$$
\mathbb{Z}\left[\operatorname{Gal}\left(\mathbb{Q}\left(\mu_{n}\right)^{+} / \mathbb{Q}\right)\right] \stackrel{\sim}{\longrightarrow} \frac{\mathbb{Z}\left[\operatorname{Gal}\left(\mathbb{Q}\left(\mu_{n}\right) / \mathbb{Q}\right)\right]}{<\sigma_{i}-\sigma_{-i} \mid \sigma_{i} \in \operatorname{Gal}\left(\mathbb{Q}\left(\mu_{n}\right) / \mathbb{Q}\right)>}
$$

if $n$ is a prime power, we obtain the following useful

Lemma 2.3. Under the same assumption of Lemma 2.2 we have

$$
\operatorname{ann}_{\mathbb{Z}\left[\operatorname{Gal}\left(\mathbb{Q}\left(\mu_{n}\right) / \mathbb{Q}\right)\right]} D_{n} /\left(D_{n}\right)_{\text {tor }}=\left\{\begin{array}{l}
<\sigma_{i}-\sigma_{-i} \mid \sigma_{i} \in \operatorname{Gal}\left(\mathbb{Q}\left(\mu_{n}\right) / \mathbb{Q}\right)> \\
\text { if } n \text { is a prime power, } \\
<\sigma_{i}-\sigma_{-i} \mid \sigma_{i} \in \operatorname{Gal}\left(\mathbb{Q}\left(\mu_{n}\right) / \mathbb{Q}\right), N_{+}> \\
\text {otherwise. }
\end{array}\right.
$$

The torsion $\left(D_{n}\right)_{\text {tor }}$ of $D_{n}$ can be easily determined. We know that $\left(D_{n}\right)_{\text {tor }}$ is contained in the torsion of the unit group of $\mathbb{Q}\left(\mu_{n}\right)$ which is $\pm \mu_{n}$. The torsion $\left(D_{n}\right)_{\text {tor }}$ contains $\pm \zeta_{n}$ as $\left(\sigma_{1}-\sigma_{-1}\right)\left(1-\zeta_{n}\right)=-\zeta_{n}$ and thus we have

$$
\left(D_{n}\right)_{\mathrm{tor}}= \begin{cases}\mu_{n} & \text { if } n \text { is even } \\ \pm \mu_{n} & \text { if } n \text { is odd. }\end{cases}
$$

In the argument of the proof of Theorem A we need to compute the annihilators of $D_{n}$ explicitly.

Proposition 2.4. Let $p$ be an odd prime and $n=4 p$. Then the annihilators $\mathcal{A}(n)$ of $D_{n}$ in the group ring $R_{n}$ are generated over $\mathbb{Z}$ by the following sets,

$$
\begin{aligned}
& \left\{n\left(\sigma_{1}-\sigma_{-1}\right), k\left(\sigma_{1}-\sigma_{-1}\right)-\left(\sigma_{k}-\sigma_{-k}\right),\right. \\
& \left.\sum_{\sigma \in G\left(\mathbb{Q}\left(\mu_{n}\right) / \mathbb{Q}\left(\mu_{4}\right)\right)} \sigma \mid 3 \leq k \leq n / 2,(k, n)=1\right\}
\end{aligned}
$$

if $p \equiv 1$ modulo 4 ,

$$
\begin{aligned}
& \left\{\frac{n}{4}\left(\sigma_{1}-\sigma_{-1}\right)-N_{+}(n), k\left(\sigma_{1}-\sigma_{-1}\right)-\left(\sigma_{k}-\sigma_{-k}\right),\right. \\
& \left.\sum_{\sigma \in G\left(\mathbb{Q}\left(\mu_{n}\right) / \mathbb{Q}\right)} \sigma \mid 3 \leq k \leq n / 2,(k, n)=1\right\}
\end{aligned}
$$

if $p \equiv 3$ modulo 4 . 
Proof. Let $\rho_{n}$ be the map from $R_{n}$ to $D_{n}$ defined by $\rho_{n}(a)=\left(1-\zeta_{n}\right)^{a}$. Let pr be the natural projection from $D_{n}$ to $D_{n} / \mu_{n}$. Let $\bar{\rho}_{n}:=\operatorname{pr} \circ \rho_{n}$ be the map from $R_{n}$ to $D_{n} / \mu_{n}$. Let $\mathcal{A}_{n}:=\operatorname{Ker}\left(\bar{\rho}_{n}\right)$. Note that $\mathcal{A}(n)=\operatorname{Ker}\left(\rho_{n}\right)=\operatorname{ann}\left(1-\zeta_{n}\right)$. By applying the snake lemma to the following diagram,

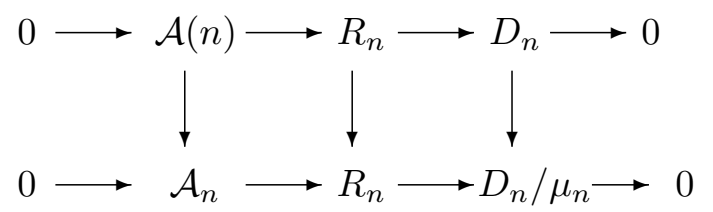

we see that the cokernel of the map from $\mathcal{A}(n)$ to $\mathcal{A}_{n}$ has order $n$. We know the $\mathbb{Z}$-basis of $\mathcal{A}_{n}$ from Lemma 2.3. Hence in order to find $\mathbb{Z}$ basis of $\mathcal{A}(n)$ it is enough to find a $\mathbb{Z}$-independent set in $\mathcal{A}(n)$ such that the relative volume(index) is $n$. It also follows from Lemma 2.2 that $D(n) / \mu_{n}$ and $R_{n}$ are free $\mathbb{Z}$-modules of rank $\varphi(n) / 2-1, \varphi(n)$ respectively. Throughout the proof of the proposition we will identify $N_{+}(n)$ with an element in $\mathbb{Z}\left[\operatorname{Gal}\left(\mathbb{Q}\left(\mu_{n}\right) / \mathbb{Q}\left(\mu_{4}\right)\right)\right]$ so that $N_{+}(n)=$ $\sum_{\sigma \in G\left(\mathbb{Q}\left(\mu_{n}\right) / \mathbb{Q}\left(\mu_{4}\right)\right)} \sigma$ via the following isomorphisms,

$$
\operatorname{Gal}\left(\mathbb{Q}\left(\mu_{n}\right) / \mathbb{Q}\left(\mu_{4}\right)\right) \simeq \operatorname{Gal}\left(\mathbb{Q}\left(\mu_{p}\right) / \mathbb{Q}\right) \simeq \operatorname{Gal}\left(\mathbb{Q}\left(\mu_{n}\right)^{+} / \mathbb{Q}\right) .
$$

First suppose that $p \equiv 1$ modulo 4 . From Lemma 2.3, we set the following $\mathbb{Z}$-basis of $\mathcal{A}_{n}$ :

$$
v_{i}:= \begin{cases}\sigma_{i}-\sigma_{-i} & \text { if } 1 \leq i \leq n / 2, \quad(i, n)=1 \\ N_{+}(n) & \text { if } i=\phi(n) / 2+1\end{cases}
$$

We define the following $\mathbb{Z}$-independent set in the group ring $R_{n}$.

$$
w_{i}:= \begin{cases}n\left(\sigma_{1}-\sigma_{-1}\right) & \text { if } i=1, \\ i\left(\sigma_{1}-\sigma_{-1}\right)-\left(\sigma_{i}-\sigma_{-i}\right) & \text { if } 3 \leq i \leq n / 2, \quad(i, n)=1, \\ N_{+}(n) & \text { if } i=\phi(n) / 2+1 .\end{cases}
$$

The set defined above is actually contained in $\mathcal{A}(n)$, the annihilators of $D_{n}$, which can be easily shown. For instance the last term $N_{+}(n)$ can be checked as follows,

$$
N_{+}(n)\left(1-\zeta_{p} \zeta_{4}\right)=\left(1-\zeta_{4}\right)^{\operatorname{Fr}_{p}-1}=1 .
$$

In order to compute the relative volume of two spaces generated by $v_{i}$ 's and $w_{i}$ 's we write $w_{i}$ in terms of $v_{j}, w_{i}=\sum a_{i j} v_{j}$. The $p \times p$ matrix $\left(a_{i j}\right)$ can be written in the following form. 


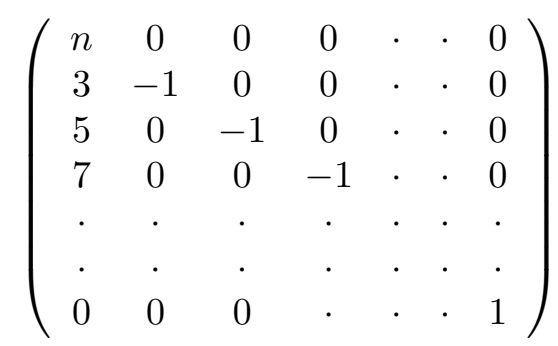

Then it follows that the determinant of this matrix is equal to $-n$ which proves the first half of the proposition.

Next suppose that $p \equiv 3$ modulo 4 . We write $p=4 k+3$. From the isomorphisms of Galois groups above we extend $\sigma_{i} \in \operatorname{Gal}\left(\mathbb{Q}\left(\mu_{p}\right) / \mathbb{Q}\right)$ defined to be $\sigma_{i}\left(\zeta_{p}\right)=\zeta_{p}^{i}$ into $\sigma_{x_{i}} \in \operatorname{Gal}\left(\mathbb{Q}\left(\mu_{4 p}\right) / \mathbb{Q}\left(\mu_{4}\right)\right)$ with $\sigma_{x_{i}}\left(\zeta_{n}\right)=\zeta_{n}^{x_{i}}$ and $\sigma_{x_{i}}\left(\zeta_{4}\right)=\zeta_{4}$. We have the following congruence relation

$$
x_{i} \equiv\left\{\begin{array}{cc}
i & \text { modulo } p \\
1 & \text { modulo } 4 .
\end{array}\right.
$$

Hence $x_{i}$ satisfies

$$
x_{i} \equiv-p+4(k+1) i \text { modulo } n .
$$

We associate to each $x_{i}$ a unique integer $\bar{x}_{i} \in \mathbb{Z}$ which lies between $-2 p$ and $2 p$ and congruent to $x_{i}$ modulo $n$. Let $X_{+}$denote the set of $x_{i}$ 's such that $0<\bar{x}_{i}<2 p$. Similarly $X_{-}$for those $x_{i}$ with $-2 p<\bar{x}_{i} \leq 0$ :

$$
X_{+}=\left\{x_{i} \mid 1 \leq i \leq p-1, \quad 0<\bar{x}_{i}<2 p\right\}
$$

and

$$
X_{-}=\left\{x_{i} \mid 1 \leq i \leq p-1, \quad-2 p<\bar{x}_{i} \leq 0\right\} .
$$

In order to compute $X_{+}$and $X_{-}$explicitly using the above congruence $x_{i} \equiv$ $-p+4(k+1) i$ modulo $n$ we need to find indices $i^{\prime} s$ such that the following equalities hold for some integers $t$ and $s$,

$$
-2 p<p(i-1)+i+n t \leq 0,0<p(i-1)+i+n s<2 p .
$$

We will need to define the following partitions of $S_{p-1}:=\{1, \ldots, p-1\}$,

$$
\text { for } r=1,2, \quad S_{p-1}(r):=\{4 l+r \mid 0 \leq l \leq k\}
$$

and

$$
\text { for } r=3,4, \quad S_{p-1}(r):=\{4 l+r \mid 0 \leq l \leq k-1\} .
$$

By pluging each index $i$ chosen from $S_{p-1}(r)$ 's into the above congruence $(*)$, $X_{+}$and $X_{-}$can be found as follows,

$$
\begin{aligned}
& X_{+}=\left\{x_{a} \mid a \in S_{p-1}(1) \cup S_{p-1}(2)\right\}, \\
& X_{-}=\left\{x_{a} \mid a \in S_{p-1}(3) \cup S_{p-1}(4)\right\} .
\end{aligned}
$$

Note that the cardinality $\left|X_{+}\right|$of $X_{+}$is equal to $2 k+2$ and $\left|X_{-}\right|=2 k$. We let

$$
\Delta\left(X_{-}\right):=\sum_{i \in X_{-}} \bar{x}_{i}=\sum_{l=0}^{k-1}\left(\bar{x}_{4 l+3}+\bar{x}_{4 l+4}\right) .
$$


When $l=0$ in order to get $\bar{x}_{3}$ and $\bar{x}_{4}$ one must subtract $n$ from $x_{3}$ and $x_{4}$ respectively. In the same way to get $\bar{x}_{4 l+3}$ and $\bar{x}_{4 l+4}$ one need to subtract $n(l+1)$ respectively. It follows from $x_{i} \equiv-p+4(k+1) i$ that the sum $\Delta\left(X_{-}\right)$ becomes

$$
\Delta\left(X_{-}\right)=\sum_{l=0}^{k-1}(-2 p+4(k+1)(8 l+7)-8 p(l+1))=-2 p k
$$

This sum $\Delta\left(X_{-}\right)$will be used to compute the determinant of the relative matrix later. From Lemma 2.3, we set the following $\mathbb{Z}$-basis of $\mathcal{A}_{n}$,

$$
v_{i}:= \begin{cases}\sigma_{i}-\sigma_{-i} & \text { if } 1 \leq i \leq n / 2, \quad(i, n)=1, \\ N_{+}(n) & \text { if } i=\phi(n) / 2+1 .\end{cases}
$$

We define the following $\mathbb{Z}$-independent set in the group ring $R_{n}$,

$$
w_{i}:= \begin{cases}\frac{n}{4}\left(\sigma_{1}-\sigma_{-1}\right)-N_{+}(n) & \text { if } i=1, \\ i\left(\sigma_{1}-\sigma_{-1}\right)-\left(\sigma_{i}-\sigma_{-i}\right) & \text { if } 3 \leq i \leq n / 2, \quad(i, n)=1, \\ \sum_{\sigma \in \operatorname{Gal}\left(\mathbb{Q}\left(\mu_{n}\right) / \mathbb{Q}\right)} \sigma & \text { if } i=\frac{\phi(n)}{2}+1 .\end{cases}
$$

We claim that these elements $w_{i}$ 's are contained in $\mathcal{A}(n)$. As $N_{+}(n)\left(1-\zeta_{4} \zeta_{p}\right)$ is equal to $\left(1-\zeta_{4}\right)^{F r_{p}-1}=-\zeta_{4}^{-1}$ it follows that $w_{1}=p\left(\sigma_{1}-\sigma_{-1}\right)-N_{+}(n)$ annihilates $D_{n}$. For $3 \leq i \leq n / 2, \quad(i, n)=1$, it is obvious that $w_{i}$ kills $1-$ $\zeta_{n}$. As $n$ is not a prime power it follows that the norm $\sum_{\sigma \in \operatorname{Gal}\left(\mathbb{Q}\left(\mu_{n}\right) / \mathbb{Q}\right)} \sigma$ also annihilates $1-\zeta_{n}$. This justifies our claim. Now we express the norm $N(n):=$ $\sum_{\sigma \in \operatorname{Gal}\left(\mathbb{Q}\left(\mu_{n}\right) / \mathbb{Q}\right)} \sigma$ in terms of $N_{+}(n)$ and $v_{1}, \ldots, v_{2 p-1}$,

$$
\begin{aligned}
N(n) & =2 N_{+}(n)+\sum_{i \in X_{+}} v_{i}+\sum_{i \in X_{-}} v_{-i} \\
& =2 N_{+}(n)+\sum_{i \in X_{+}} v_{i}-\sum_{i \in X_{-}} v_{i}
\end{aligned}
$$

We define $\operatorname{sgn}(i)$ to be

$$
\operatorname{sgn}(i):=\left\{\begin{array}{lll}
1 & \text { if } & \bar{x}_{i} \in X_{+} \\
-1 & \text { if } & \bar{x}_{i} \in X_{-}
\end{array}\right.
$$

Note that $w_{\frac{\phi(n)}{2}+1}$ is written in the following form.

$$
w_{\frac{\phi(n)}{2}+1}=\sum_{\sigma \in G} \sigma=2 N_{+}(n)+\sum \operatorname{sgn}(i) v_{i} .
$$

Again in order to compute the relative volume of two spaces generated by $v_{i}$ 's and $w_{i}$ 's we write $w_{i}$ in terms of $v_{j}, w_{i}=\sum a_{i j} v_{j}$. The $p \times p$ matrix $\left(a_{i j}\right)$ can be written in the following form. 


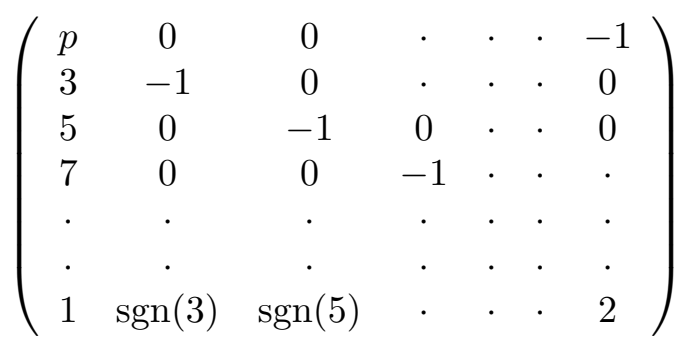

We compute the determinant of this matrix in the following equation.

$$
\begin{aligned}
\operatorname{det}\left(a_{i j}\right) & =-2 p-\left(\sum_{1 \leq l \leq 2 p-1,(l, 4 p)=1} \operatorname{sgn}(l) l\right) \\
& =-2 p-\left(\sum_{1 \leq l \leq 2 p-1,(l, 4 p)=1} l-2\left(\sum_{1 \leq l \leq 2 p-1,(l, 4 p)=1, \operatorname{sgn}(l)=-1} l\right)\right) \\
& =-2 p-\left(\left(p^{2}-p\right)+2 \Delta\left(X_{-}\right)\right) \\
& =-2 p-\left(\left(p^{2}-p\right)+2(-2 p k)\right) \\
& =-2 p-\left(p^{2}-p-p(p-3)\right) \\
& =-4 p=-n
\end{aligned}
$$

This completes the proof of the Proposition.

We are ready to prove the following theorem that $\Psi$ is a free $\mathbb{Z}$-module.

Theorem 2.5. $\Psi_{\text {tor }}=1$.

Proof. Let $f$ be a nontrivial element of $\Psi_{\text {tor }}$. By Theorem 2.1, $f=\delta_{\text {odd }}$. Let $d_{n}:=n\left(\sigma_{1}-\sigma_{-1}\right) \in R_{n}$. One can see that $\delta_{\text {odd }}\left(\zeta_{n}\right)=\psi_{d_{n}}\left(\zeta_{n}\right)$ for all $n$. As $f \in \Psi_{\text {tor }}, f$ can be written $f=\psi_{r}$ for some $r=\left(r_{n}\right) \in R$. Let $p$ be a fixed odd prime and let $n=4 p$. It follows from $\delta_{\text {odd }}\left(\zeta_{4 p}\right)=\psi_{d_{4 p}}\left(\zeta_{4 p}\right)=f\left(\zeta_{4 p}\right)=\psi_{r}\left(\zeta_{4 p}\right)$ that

$$
r_{4 p}=d_{4 p}+a_{4 p}, \text { for some } a_{4 p} \in \operatorname{ann}_{R_{4 p}}\left(1-\zeta_{4 p}\right) .
$$

We observe the following equation.

$$
\begin{aligned}
-1=\delta_{\text {odd }}\left(\zeta_{p}\right) & =\psi_{d_{p}}\left(\zeta_{p}\right)=\left(1-\zeta_{p}\right)^{p\left(\sigma_{1}-\sigma_{-1}\right)} \\
& =\left(1-\zeta_{p}\right)^{d_{p}}=\left(1-\zeta_{p}\right)^{r_{p}} \\
& =\left(1-\zeta_{p}\right)^{r_{4 p}}=\left(1-\zeta_{p}\right)^{d_{4 p}+a_{4 p}}, \text { as } r=\left(r_{n}\right) \in R \\
& =\left(1-\zeta_{p}\right)^{a_{4 p}}
\end{aligned}
$$

We define the following notations.

$$
\xi_{i}^{j}(n):=\frac{1-\zeta_{n}^{j}}{1-\zeta_{n}^{i}}, \quad \xi^{i}(n):=1-\zeta_{n}^{i} \text { for } i, j, n \in \mathbb{Z} .
$$

We show that the equation (1) is impossible for some $n$. We need the following statement.

Claim. $D_{28}=C_{28}$. 
Proof of the claim. We know that $C_{28}$ is generated by $\xi^{i}(28), \pm \zeta_{28}, \xi_{1}^{j}(7), j=$ $2,3,4,5,6$ and $\xi_{1}^{3}(4)$. By applying $\sigma_{1}-\sigma_{-1}$ to $\left(1-\zeta_{28}\right)$ we see that $D_{28}$ contains $-\zeta_{28}$ and hence $\left(-\zeta_{28}\right)^{14}=-1$. By applying the norm map $N_{28,7}$ to $\xi^{1}(28)$ we get $\xi_{1}^{4}(7)$ and $\sigma_{4} \xi_{1}^{4}(7)=\xi_{4}^{2}(7), \xi_{1}^{4}(7) \xi_{4}^{2}(7)=\xi_{1}^{2}(7)$. It follows from

$$
\sigma_{3} \xi_{1}^{4}(7)=\xi_{3}^{5}(7), \quad \sigma_{3} \xi_{1}^{2}(7)=\xi_{3}^{6}(7)=\xi_{3}^{-1}(7)=\left(-\zeta_{7}^{-1}\right) \xi_{3}^{1}(7)
$$

that $D_{28}$ contains $\xi_{3}^{1}(7)$. Furthermore it follows from

$$
\xi_{6}^{3}(7) \xi_{3}^{5}(7)=\xi_{6}^{5}(7)=\left(-\zeta_{7}\right) \xi_{1}^{5}(7)
$$

that $D_{28}$ contains $\xi_{1}^{5}(7)$. Note that $\xi_{1}^{6}(7)=\xi_{1}^{-1}(7)=-\zeta_{7}^{-1} \in D_{28}$. This shows that $D_{28}$ contains $C_{7}$. One can show that $D_{28}$ contains $C_{4}$ as well.

We apply Proposition 2.4 to find $\mathbb{Z}$-independent set in $\mathcal{A}(28)$ :

$$
w_{i}:= \begin{cases}7\left(\sigma_{1}-\sigma_{-1}\right)-N_{+} & \text {if } i=1, \\ i\left(\sigma_{1}-\sigma_{-1}\right)-\left(\sigma_{i}-\sigma_{-i}\right) & \text { if } 3 \leq i \leq 14, \quad(i, 28)=1, \\ \sum_{\sigma \in G} \sigma & \text { if } i=7 .\end{cases}
$$

It follows that if $a_{28} \in A(28)$ then $\left(1-\zeta_{7}\right)^{a_{28}}$ is a product of either $-p, p^{2}$ or 1 . Thus the equation (1) is impossible for $p=7$. Hence $\Psi_{\text {tor }}$ must be the trivial group. This completes the proof of Theorem 2.5.

As an immediate corollary of Theorem 2.1 and Theorem 2.2, we obtain the following theorem.

Theorem 2.6 (=Theorem A). $\mathcal{F} \neq \Psi$.

In the proof of Theorem 2.1 we note the following

Corollary 2.7. $\delta_{\text {odd }}$ can be written in the form of $\psi_{\left(d_{n}\right)}, d_{n} \in R_{n} ; \delta_{\text {odd }}\left(\zeta_{n}\right)=$ $\left(1-\zeta_{n}\right)^{d_{n}}$, for some $d_{n} \in R_{n}$.

Corollary 2.7 shows that for $f \in \mathcal{F}$ even if $f\left(\zeta_{n}\right)$ belongs to $\left(1-\zeta_{n}\right)^{R_{n}}$ for all $n, f$ need not belong to $\Psi$.

\section{Coherence sequences over $\mathbb{Z}_{p}$ extensions}

We are interested in characterizing norm coherence circular $p$-units in $\mathbb{Z}_{p^{-}}$ extensions. Let $a(n):=n\left(\sigma_{1}-\sigma_{-1}\right)$. Let $f_{\text {odd }}\left(\zeta_{n}\right):=\left(1-\zeta_{n}\right)^{a(n)}$. We know that $f_{\text {odd }}$ is not contained in $R$-cyclic module $\Psi$. However if we restrict $f_{\text {odd }}$ to the basic $\mathbb{Z}_{p}$ extension $\mathbb{Q}\left(\mu_{p^{\infty}}\right):=\bigcup_{n} \mathbb{Q}\left(\mu_{p^{n}}\right)$ of $\mathbb{Q}\left(\mu_{p}\right)$ (cf. [3]), then we have a nice expression of $f_{\text {odd }}$. We know from Lemma 2.3 that

$$
\operatorname{ann}_{\mathbb{Z}\left[\operatorname{Gal}\left(\mathbb{Q}\left(\mu_{p^{n}}\right) / \mathbb{Q}\right)\right]} D_{n} /\left(D_{p^{n}}\right)_{\text {tor }}=<\sigma_{i}-\sigma_{-i}: \sigma_{i} \in \operatorname{Gal}\left(\mathbb{Q}\left(\mu_{p^{n}}\right) / \mathbb{Q}\right)>.
$$

Using the same arguments of Proposition 2.4 we can obtain

(2) $\mathcal{A}_{p^{n}}:=\operatorname{ann}_{\mathbb{Z}\left[\operatorname{Gal}\left(\mathbb{Q}\left(\mu_{p^{n}}\right) / \mathbb{Q}\right)\right]} D_{p^{n}}=$

$<4\left(\sigma_{1}-\sigma_{-1}\right)-2\left(\sigma_{2}-\sigma_{-2}\right), k\left(\sigma_{1}-\sigma_{-1}\right)-\left(\sigma_{k}-\sigma_{-k}\right): 3 \leq i \leq p^{n} / 2,(i, p)=1>$ 
It follows from the coherency property ( for $m>n$,)

$$
\operatorname{res}\left(a\left(p^{m}\right)\right) \equiv a\left(p^{n}\right) \text { modulo } \operatorname{ann}_{R_{p^{n}}} D_{p^{n}}
$$

that

$$
\operatorname{res}\left(a\left(p^{m}\right)\right)=a\left(p^{n}\right)+\alpha\left(p^{n}\right), \text { for some } \alpha\left(p^{n}\right) \in \operatorname{ann}_{R_{p^{n}}} D_{p^{n}} .
$$

We can lift the element $\alpha\left(p^{n}\right)$ to $\widehat{\alpha}\left(p^{n}\right)$ in $R_{p^{m}}$ which is possible from the equation (2) of the annihilators $\operatorname{ann}_{R_{p^{n}}} D_{n}$. If we reset $\widehat{a}\left(p^{m}\right):=a\left(p^{m}\right)-\widehat{\alpha}\left(p^{n}\right)$ instead of $a\left(p^{m}\right)$, then we have the following equation:

$$
f_{\text {odd }}\left(\zeta_{p^{n}}\right)=\left(1-\zeta_{p^{n}}\right)^{\widehat{a}\left(p^{n}\right)}, \widehat{a}\left(p^{n}\right) \in R_{p^{\infty}}\left(:=\lim _{\longleftarrow} R_{p^{n}}\right) .
$$

We believe that this happens for the coherence sequence $f_{\text {odd }}\left(\zeta_{p^{n}}\right)=((-1))_{p^{m}}$ comes from values of a universal circular distribution $f_{\text {odd }} \in \mathcal{F}$. From this observation we are tempted to state the following guess. (Note that this is weaker than Coleman's guess.)

Guess. Let $\left(a_{p^{n}}\right)$ be a norm coherence sequence over the basic $\mathbb{Z}_{p^{-}}$-extension $\mathbb{Q}\left(\mu_{p^{\infty}}\right)$. Then $a_{p^{n}}$ is a norm coherence sequence of circular $p$-units if and only if there exists an $f \in \mathcal{F}$ such that $f\left(\zeta_{p^{n}}\right)=a_{p^{n}}$ for all $n$.

Let $\left\{G_{n}\right\}$ be an inverse system of abelian groups with homomorphisms $r_{m, n}$ : $G_{m} \longrightarrow G_{n}$. Let $H_{n}$ be a subgroup of $G_{n}$. Let $\Omega\left(G_{n}, H_{n}\right):=<h-1: h \in H_{n}>$ be the $\mathbb{Z}\left[G_{n}\right]$ submodule generated by $h-1, h \in H_{n}$. It follows from the exact sequence

$$
1 \longrightarrow \Omega\left(G_{n}, H_{n}\right) \longrightarrow \mathbb{Z}\left[G_{n}\right] \longrightarrow \mathbb{Z}\left[G_{n} / H_{n}\right] \longrightarrow 1
$$

that

$$
1 \longrightarrow \lim _{\longleftarrow} \Omega\left(G_{n}, H_{n}\right) \longrightarrow \lim _{\longleftarrow} \mathbb{Z}\left[G_{n}\right] \longrightarrow \lim _{\longleftarrow} \mathbb{Z}\left[G_{n} / H_{n}\right]
$$

If $r_{m, n}: \Omega\left(G_{m}, H_{m}\right) \longrightarrow \Omega\left(G_{n}, H_{n}\right)$ is surjective for all sufficiently large $n, m$ with $n \leq m$ then we have the exact sequence

$$
1 \longrightarrow \lim _{\longleftarrow} \Omega\left(G_{n}, H_{n}\right) \longrightarrow \lim _{\longleftarrow} \mathbb{Z}\left[G_{n}\right] \longrightarrow \lim _{\longleftarrow} \mathbb{Z}\left[G_{n} / H_{n}\right] \longrightarrow 1 \text {. }
$$

This is the case when $G_{n}$ is the Galois group $\operatorname{Gal}\left(\mathbb{Q}\left(\mu_{n}\right) / \mathbb{Q}\right)$ and $H_{n}$ is the $p$-complement subgroup of $G_{n}, H_{n}:=\operatorname{Gal}\left(\mathbb{Q}\left(\mu_{n / p^{e_{n}}}\right) / \mathbb{Q}\right)$, where $n=\prod p^{e_{n}}$. Hence it follows that the "only if" part of the guess holds trivially.

For the "if" part of the guess we can state that for all $f \in \mathcal{F}$ and for all $n \in \mathbb{N}$, there is a constant $c$ which depends only on $p$ such that $f^{c}\left(\zeta_{p^{n}}\right)$ is a norm coherence circular $p$-units.

Notice that all coherence sequences in $\mathbb{Z}_{p}$-tower do not have to be written in this way. A sufficient condition regard this was made by Coleman[2] in terms of Archimedean characterization: A subset $S \in \overline{\mathbb{Q}}$ is called bounded if the set $\bigcup \sigma S(\sigma$ ranging over all embeddings $\overline{\mathbb{Q}} \hookrightarrow \mathbb{C})$ is a bounded subset of $\mathbb{C}$. The following theorem is part (a) of Theorem $\mathrm{A}$ in [2].

Theorem 3.1 (Coleman). Suppose $a_{m} \in \mathbb{Q}\left(\mu_{p^{m}}\right)(m \geq 1)$ is a bounded norm compatible sequence of cyclotomic numbers. Then $a_{m}$ is an integral circular p-units for all $m \geq 1$. 
Certainly not all coherence sequence of circular units are bounded. We refer the reader to [2] for the detail. We propose a sufficient condition to the guess above. Using Theorem A in [8], we can define $w: \mathcal{F} \times P \longrightarrow \mathbb{N}: w(f, p)$ is the smallest positive integer $c$ such that $f^{c}\left(\zeta_{p^{n}}\right) \in C_{p^{n}}$, for all $n \in \mathbb{N}$. Using the equation (2) we also define $\gamma: \mathcal{F} \times P \longrightarrow \lim \left(R_{p^{n}} / \mathcal{A}_{p^{n}}\right): f^{w(f, p)}\left(\zeta_{p^{n}}\right)=$ $\psi_{\gamma(f, p)}\left(\zeta_{p^{n}}\right)$. Note that $\gamma(f, p)$ has a lifting to $R_{p^{\infty}}$ from the equation (2). Let $\mathbb{Z}_{+}, \mathbb{Z}_{-}$be the set of positive integers and set of negative integers respectively. Let $R_{p^{\infty}}^{+}$be the subset of $R_{p^{\infty}}$ with positive integral coefficients:

$$
R_{p^{\infty}}^{+}:=\left\{\left(r_{p^{n}}\right) \in R_{p^{\infty}} \mid r_{p^{n}} \in \mathbb{Z}_{+}[G] \text { for all } n \in \mathbb{N}\right\}
$$

and similarly

$$
R_{p^{\infty}}^{-}:=\left\{\left(r_{p^{n}}\right) \in R_{p^{\infty}} \mid r_{p^{n}} \in \mathbb{Z}_{-}[G] \text { for all } n \in \mathbb{N}\right\} .
$$

Proposition 3.2. Let $f \in \mathcal{F}$ and $p$ be an odd prime. Suppose that $\gamma(f, p)$ has a lifting to $\widehat{\gamma}(f, p) \in R_{p \infty}$ such that

$$
\widehat{\gamma}(f, p)=\widehat{\gamma}^{+}(f, p)+\widehat{\gamma}^{-}(f, p),
$$

for some $\widehat{\gamma}^{+}(f, p) \in R_{p^{\infty}}^{+}, \widehat{\gamma}^{-}(f, p) \in R_{p^{\infty}}^{-}$. Then $f\left(\zeta_{p^{n}}\right)=\psi_{r_{p^{n}}}\left(\zeta_{p^{n}}\right)$ for some $\left(r_{p^{n}}\right) \in R_{p^{\infty}}$.

Proof. From the assumption, there is a constant $w$ such that

$$
f\left(\zeta_{p^{n}}\right)^{w}=\psi_{\widehat{\gamma}^{+}(f, p)}\left(\zeta_{p^{n}}\right) \psi_{\widehat{\gamma}^{-}(f, p)}\left(\zeta_{p^{n}}\right) .
$$

It follows that

$$
\left(\psi_{\widehat{\gamma}^{-}(f, p)}^{-1}\left(\zeta_{p^{n}}\right) f\left(\zeta_{p^{n}}\right)\right)^{w}=\psi_{\widehat{\gamma}^{+}(f, p) \widehat{\gamma}^{-}(f, p)^{-w}}\left(\zeta_{p^{n}}\right) .
$$

The right hand side of the above equation is a bounded norm coherence sequence. Thus $\psi_{\widehat{\gamma}^{-}(f, p)}^{-1}\left(\zeta_{p^{n}}\right) f\left(\zeta_{p^{n}}\right)$ is a bounded norm coherence sequence. By applying Theorem 3.1 of Coleman we complete the proof.

\section{Acknowledgements}

We would like to thank the referee for helpful comments and suggestions.

\section{References}

[1] R. Coleman, Division values in local fields, Invent. Math. 53 (1979) 91-116.

[2] _ On an Archimedean characterization of the circular units, J. Reine Angew. Math. 356 (1985) 161-173.

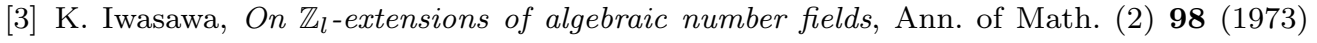
$246-326$.

[4] V. A. Kolyvagin, Euler systems, The Grothendieck Festschrift, Vol. II, 435-483, Progr. Math. 87, Birkhuser Boston, Boston, MA, 1990.

[5] B. Mazur and K. Rubin, Kolyvagin systems, Mem. Amer. Math. Soc. 168 (2004), no. 799.

[6] K. Rubin, Global units and ideal class groups, Invent. Math. 89 (1987) 511-526.

[7] _ Euler Systems, Annals of Mathematics Studies 147, Princeton University Press, 2000.

[8] S. Seo, Circular distributions and Euler systems, J. Number Theory 88 (2001) 366-379. 
[9] Circular distributions and Euler Systems II, Compositio Math. 137 (2003), 9198.

[10] _ A note on circular distributions, Acta Arith. 114 (2004) 313-322.

[11] _ Euler systems and special units, J. Number Theory 109 (2004) 59-68.

[12] W. Sinnott, On the Stickelberger ideal and the circular units of a cyclotomic field, Ann. of Math. (2) 108 (1978) 107-134.

Department of Mathematics, Yonsei University, 134 Sinchon-Dong, Seodaemun-Gu, Seoul 120-749, South Korea

E-mail address: sgseo@yonsei.ac.kr 